\title{
Serum concentrations of the interferon- $\gamma$-inducible chemokine IP-10/CXCL10 are augmented in both newly diagnosed Type I diabetes mellitus patients and subjects at risk of developing the disease
}

\author{
F. Nicoletti ${ }^{1}$, I. Conget ${ }^{2}$, M. Di Mauro ${ }^{1}$, R. Di Marco ${ }^{3}$, M. C. Mazzarino¹, K. Bendtzen ${ }^{4}$, A. Messina ${ }^{1}$, R. Gomis ${ }^{2}$ \\ ${ }^{1}$ Department of Biomedical Sciences, University of Catania, Italy \\ ${ }^{2}$ Endocrinology and Diabetes Unit, Hospital Clinic, Faculty of Medicine, University of Barcelona, IDIBAPS, Barcelona, Spain \\ ${ }^{3}$ Department of Microbiological and Gynaecological Sciences, University of Catania, Catania, Italy \\ ${ }^{4}$ Institute for Inflammation Research, Rigshospitalet University Hospital, Copenhagen, Denmark
}

\section{Abstract}

Aims/hypothesis. Chemokines are chemotactic cytokines controlling the recruitment of leukocytes from the blood by regulating integrin adhesiveness. It has been shown that the migration of CD4+Th1 and CD4+Th2 cells is governed by specific chemokines.

Increasing evidence suggests that the CD4+Th1 cheomoattractant chemokine CXCL10, also termed Interferon (IFN)- $\gamma$-inducible protein (IP)-10 is pathogenetically involved in several immunoinflammatory and autoimmune diseases.

Methods. IFN- $\gamma$ and IP-10 were quantified by solidphase ELISA in sera of patients with either newly diagnosed or long-term Type I (insulin-dependent) diabetes mellitus, and in sera of their healthy first degree relatives. The latter were subdivided into "low" and "high" risk prediabetic subjects depending on whether they were negative or positive for the anti-beta-cell autoantibodies ICA and GAD.

Results. Compared with healthy control subjects $(18 \%, 9 / 50)$, those with a low risk of disease $(21 \%$, $5 / 24$ ) and the group of patients with long-term Type I diabetes $(24 \%, 12 / 50)$, IP-10 was found more frequently and at increased concentrations in both newly diagnosed Type I diabetic patients $(84 \%, 42 / 50)$ and in those with a high risk of disease $(73 \%, 16 / 22)$; in the latter, the IP-10 concentrations correlated with those of IFN- $\gamma$.

Conclusion/interpretation. Circulating IP-10 concentrations is increased in patients with Type I diabetes, but only during the early and subclinical stage of the disease. [Diabetologia (2002) 45:1107-1110]

Keywords Autoimmunity, chemokines, cytokines, interferon-gamma, IP-10/CXCL10.
In Type I (insulin-dependent) diabetes mellitus, the migration of immune effector cells (e.g. macrophages and $\mathrm{T}$ cells) from the bloodstream into the pancreatic islet is necessary to exert their autoreactive potential and commence the local secretion of type 1 proinflammatory cytokines such as (IFN)- $\gamma$, interleukin (IL)- $1 \beta$,

Received: 15 November 2001 / Revised: 11 March 2002

Published online: 21 June 2002

(C) Springer-Verlag 2002

Corresponding author: Dr. F. Nicoletti, Department of Biomedical Sciences, Section of General Pathology, University of Catania, Via Luigi Sturzo n.3, 95021, Cannizzaro, Aci-Castello, Catania, Italy. E-mail: ferdinic@ citiesonline.it

Abbreviations: ICA, Islet cell antibodies; IFN- $\gamma$, interferon- $\gamma$; IP-10, IFN- $\gamma$-inducible protein 10.
IL-2, IL-12, IL-18, and tumour necrosis factor (TNF)$\alpha$ [1]. The entry of leukocytes into tissue compartments is primarily controlled by adhesion molecules and chemokines [2]. Whilst several studies have clearly proven the involvement of adhesion molecules in the pathogenesis of the disease [3], less is known about the possible contribution of chemokines.

Chemokines are chemotactic cytokines controlling the recruitment of leukocytes from the blood by regulating integrin adhesiveness [2]. Depending on the position of N-terminal cysteine residues, chemokines can be divided into four highly conserved families namely CXC, CX3C ( $\alpha$ chemokines), and $\mathrm{C}$ and $\mathrm{CC}$ ( $\beta$ chemokines). It has been shown that the migration of CD4+ Th1 and CD4+ Th2 cells is governed by specific chemokines. For example, activated Th1 cells express the chemokine receptors CCR5 and CXCR3, whereas 
activated Th2 cells express CCR3, CCR4 and CCR8 [2]. The ligand for CXCR3 is CXCL10, also termed IFN- $\gamma$-inducible protein-10 (IP-10). Increasing evidence suggests a role of this chemokine in immunoinflammatory diseases, including multiple sclerosis, autoimmune hepatitis and thyroiditis $[4,5,6,7]$.

We measured serum concentrations of IP-10 in groups of newly diagnosed and long-lasting Type I diabetic patients as well as in subjects at "high" and "low" risk of developing the disease, depending on whether they were positive or negative for islet cell antibodies (ICA) and anti-glutamic acid decarboxylase $(\mathrm{GAD})$ antibodies.

\section{Materials and methods}

Subjects and blood sampling. The study groups consisted of: (i) 50 patients ( 20 women, 30 men; mean age 35 years, range 20-52 years) with long-standing Type I diabetes; (ii) 50 patients with Type I diabetes who were recruited within 6 weeks of diagnosis (30 women, 20 men; mean age 25 years, range 18-35 years); (iii) 16 women and 6 men who were relatives of Type I diabetic patients and at a "high-risk" for developing the disease (mean age 25 years, range 19-36 years) who were all normoglycaemic and did not meet any clinical or laboratory criteria for the disease; (iv) 24 subjects who were "low-risk" relatives of Type I diabetic patients ( 18 women, 6 men; mean age 26 years, range 16-35 years; and (v) 50 healthy control subjects who were matched for age and sex (mean age 28 years, range18-41 years). None of the subjects suffered from infectious, allergic or other autoimmune diseases in the 6 month period prior to sampling. None used immunomodulatory drugs in the last 3 months before the study, serum samples were also obtained [8]. The study design was approved by the local ethics committees and permission was obtained from the participants, who were informed about the purposes of the study.

IP-10/CXCL10 and IFN- $\gamma$ assays. IP-10 was measured by a solid-phase ELISA from R \& D Systems (Minneapolis, Minn., USA). A monoclonal antibody to human IP-10 was used for capture, and a biotinylated polyclonal antibody was used for detection. Recombinant human IP-10 served as standard. The lower sensitivity limit was $20 \mathrm{pg} / \mathrm{ml}$, and the intra-assay and inter-assay coefficients of variation were below $5 \%$.
IFN- $\gamma$ was measured by solid-phase ELISA according to the manufacturers recommendations (Endogen, Cambridge, Minn., USA). The lower sensitivity limit was $5 \mathrm{pg} / \mathrm{ml}$, and the intra-assay and inter-assay coefficients of variation were below $10 \%$.

To exclude the possibility that heterophilic antibodies interfered with the measurements, control experiments were carried out by adding irrelevant mouse IgG (Sigma Chimica, Milan, Italy) [8]. ICA and GAD antibodies were determined as described [8].

Statistical analysis. The two-sided Fisher's Exact test and Mann-Whitney U test were used to evaluate contingencies and quantitative differences, respectively. Correlations between serum IP-10 and IFN- $\gamma$ concentrations were analysed by the Spearman's rank correlation coefficient test.

\section{Results}

IP-10/CXCL10 and IFN- $\gamma$ blood concentrations. IP-10 was detected with comparable low frequencies (18 to $22 \%$ ) and similar concentrations in the sera of healthy control subjects, long-standing Type I diabetes patients and "low risk" subjects (Table 1, Fig. 1). In contrast, IP-10 was detected with higher frequencies (73 to $84 \%$ ) and approximately three times higher in subjects with newly diagnosed disease or at "high risk" of disease.

IFN- $\gamma$ was detected at very low frequencies and at comparable concentrations in healthy control subjects, in "low risk" subjects, and in patients with long-standing Type I diabetes mellitus (Table 1, Fig. 1). In contrast, IFN- $\gamma$ was detected in a larger proportion of newly diagnosed Type I diabetic patients and with an even higher frequency in "high risk" subjects. These groups also had higher concentrations of IFN- $\gamma$ (Table 1, Fig. 1).

In "high risk" subjects and in newly diagnosed Type I diabetic patients, where the number of both IP$10-$ and IFN- $\gamma$ positives allowed linear regression analyses, the serum concentrations of the two cytokines correlated significantly: $r=0.61 \quad(p=0.02)$ and 0.61 $(p=0.007)$, respectively.

Table 1. Serum concentrations of IP-10/CXCL10 and IFN- $\gamma$

\begin{tabular}{|c|c|c|c|c|c|}
\hline \multirow[t]{2}{*}{ Groups } & \multirow[t]{2}{*}{$n$} & \multicolumn{2}{|l|}{ IP-10/CXCL10 } & \multicolumn{2}{|l|}{$\mathrm{IFN}-\gamma$} \\
\hline & & Pos $(\%)$ & $\begin{array}{l}\text { Median pg/ml } \\
\text { (ranges) }\end{array}$ & Pos $(\%)$ & $\begin{array}{l}\text { Median pg/ml } \\
\text { (ranges) }\end{array}$ \\
\hline Control subjects & 50 & $9(18)$ & $70(30-105)$ & $2(4)$ & $14(10-18)$ \\
\hline Low risk subjects & 24 & $5(21)$ & $76(50-130)$ & $2(8)$ & $8.5(5-12)$ \\
\hline High risk subjects & 22 & $16(73) p<0.0001^{\mathrm{a}}$ & $200(116-650) p<0.0001^{b}$ & $14(64) p<0.0001^{\mathrm{a}}$ & $43.5(20-75) p=0.017^{\mathrm{b}}$ \\
\hline $\begin{array}{l}\text { Newly diagnosed Type I } \\
\text { diabetic patients }\end{array}$ & 50 & $42(84) p<0.0001^{\mathrm{a}}$ & $198(94-712) p^{<0.0001^{b}}$ & $18(36) p<0.0001^{\mathrm{a}}$ & $37(12-50)$ \\
\hline $\begin{array}{l}\text { Long standing Type I } \\
\text { diabetic patients }\end{array}$ & 50 & $12(24)$ & $58(30-83)$ & $2(4)$ & $15(7-23)$ \\
\hline
\end{tabular}

$p$ values were calculated relative to the control subjects using the two-sided Fisher's Exact test ${ }^{\mathrm{a}}$ or Mann-Whitney $\mathrm{U}$ test $\mathrm{t}^{\mathrm{b}}$ 


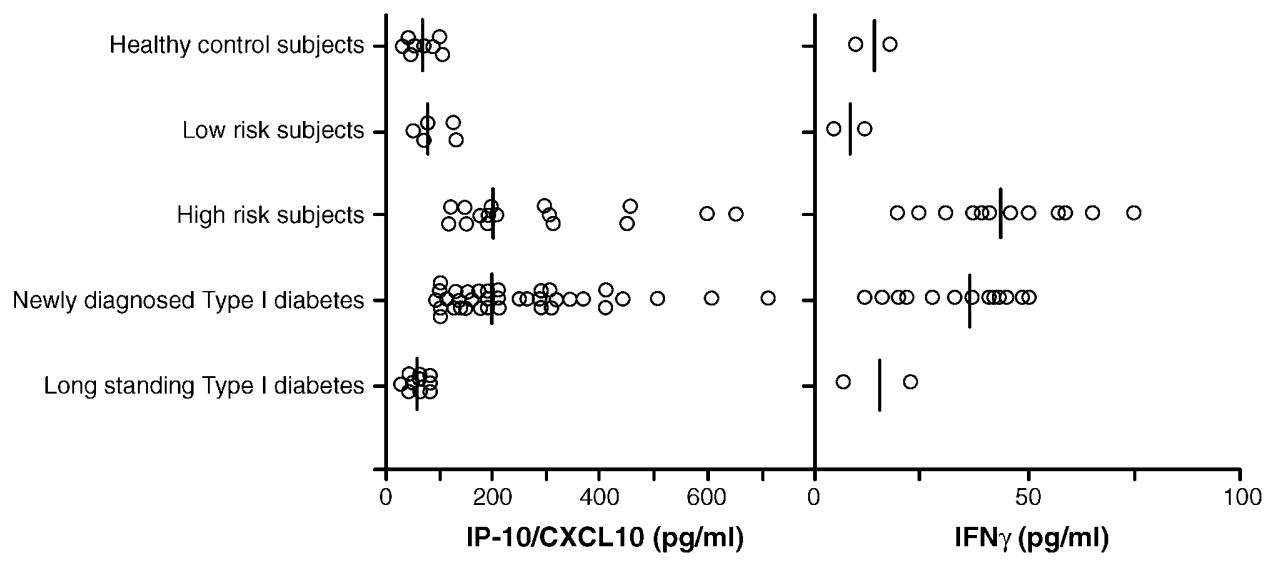

Fig. 1. Augmented blood concentrations of IP-10 in newly diagnosed Type I diabetic patients and subjects at risk of developing the disease. IFN- $\gamma$ and IP-10 were quantitated by solidphase ELISA in sera of patients with either newly diagnosed or long standing Type I diabetes mellitus, and in sera of their healthy first degree relatives. The latter were subdivided into "low" and "high" risk prediabetic subjects depending on whether they were negative or positive for the anti-beta-cell autoantibodies ICA and GAD. Sex and age-matched healthy subjects were used as controls

Addition of mouse $\operatorname{IgG}$ failed to modify the concentrations of IP-10 and IFN- $\gamma$ and also failed to change the optical densities of the negative samples.

There were no correlations to sex or age or to the frequencies and concentrations of anti-beta-cell antibodies.

\section{Discussion}

The data show increased serum concentrations of IP10/CXCL10 both in newly diagnosed Type I diabetic patients and in subjects at "high risk" of disease, and these concentrations correlated positively with those of IFN- $\gamma$. Long-lasting Type I diabetic patients and "low risk" subjects had IP-10 and IFN- $\gamma$ concentrations comparable to those found in the healthy control subjects.

We sub-divided relatives of Type I diabetic patients into groups at "high risk" of developing the disease (those positive for ICA and GAD antibodies) and those at "low risk" (those negative for both antibodies). The validity of this classification was substantiated by the observation that during a 6-year follow-up period conducted in 12 relatives of the 22 "high risk" subjects and 13 relatives of 24 "low risk" subjects, Type I diabetes developed in 8 of the 12 "high risk" relatives and in none of the "low risk" relatives.

In a recent study [9] augmented serum concentrations of IP-10 in a group of both autoantibody-positive and autoantibody-negative Type I diabetic patients have also been reported. The two studies are, however, difficult to compare, as the other study [9] did not measure IP-10 concentrations in newly diagnosed Type I diabetic patients nor in subjects at high or low risk for developing the disease. Whilst increased concentrations of IP-10 were found in the other study [9] in patients with diabetes for less than 3 years, compared to those with longer duration of diabetes, there was no specific information on the concentrations of IP-10 in the early stages of Type I diabetes. This is important when considering the tendency of many immunoinflammatory processes to decline with time, especially relevant in diseases with progressive reduction or elimination of target autoantigens. In agreement with this, we could not detect IP-10 with higher frequency or at a higher concentration in Type I diabetes patients with diabetes for more than 5 years.

The increased IP-10 concentrations both in newly diagnosed Type I diabetic patients and in subjects at "high risk" of disease could also have diagnostic and pathogenic relevance. Thus, measuring circulating IP10 it could be used clinically as a marker of emerging beta-cell (auto)immune reactivity in relatives of Type I diabetic patients. Proof of this might be obtained from larger and longitudinal studies of the prediabetic subjects.

Our findings also support the contention that an upregulated synthesis of IP-10 could take place in the early and even preclinical phases of the disease. It is not clear whether increased production might occur in the blood only, or whether the increased serum concentrations might mirror chemokine production in the pancreas. If the latter is true, the CD4+ Th1 chemoattracting and stimulatory properties of IP-10 could be pathogenetically important by attracting and stimulating diabetogenic Th1 cells to the pancreas.

The positive correlation between the circulating concentrations of IP-10 and IFN- $\gamma$ in "high risk" subjects and, even more pronounced, in patients with newly diagnosed Type I diabetes, strengthens the assumed bidirectional link in vivo between these molecules, each stimulating and synergising the synthesis or action of the other. This could contribute to the vicious circuit of immunoinflammatory reactions leading to beta-cell destruction. 
The lack of correlation between the circulating concentrations of IP-10 and those of ICA and GAD antibodies agree with previous findings of type 1 proinflammatory cytokines, such as IL-1 $\beta$, IL-2, IL-18, IFN- $\gamma$ and TNF- $\alpha$ [10], the concentrations of which are higher in newly diagnosed Type I diabetic patients and in "high risk" subjects. As cell-mediated immunity is primarily responsible for Type I diabetes development [1] and because IP-10 like most of the above cytokines stimulates cell-mediated immunity to a greater extent than humoral immunity, it is possible that IP-10 has diabetogenic potential without influencing antibody production from autoreactive B lymphocytes.

\section{References}

1. Rabinovitch A (1998) An update on cytokines in the pathogenesis of insulin-dependent diabetes mellitus. Diabetes Metab Rev 14:129-151

2. Arimilli S, Ferlin W, Solvason N, Desphande S, Howard M, Mocci S (2000) Chemokines in autoimmune diseases. Immunol Rev 177:43-51

3. Martin S, Heidenthal E, Schulte B, Rothe H, Kolb $\mathrm{H}$ (1998) Soluble forms of intercellular adhesion molecule-1 inhibit insulitis and onset of autoimmune diabetes. Diabetologia 41:1298-1303
4. Balashov KE, Rottman JB, Weiner HL, Hancock WW (1999) CCR5(+) and CXCR3(+) T cells are increased in multiple sclerosis and their ligands MIP-1alpha and IP-10 are expressed in demyelinating lesions. Proc Natl Acad Sci USA 96:6873-6878

5. Fife BT, Kennedy KJ, Paniagua MC et al. (2001) CXCL10 (IFN- $\gamma$-Inducible Protein-10) control of the encephalitogenic CD4+T cell accumulation in the central nervous system during experimental autoimmune encephalomyelitis. J Immunol 166:7617-7624

6. Nagayama K. Enomoto N. Miyasaka Y et al. (2001) Overexpression of interferon gamma-inducible protein 10 in the liver of patients with type I autoimmune hepatitis identified by suppression subtractive hybridization. Am J Gastroenterol 96:2211-2217

7. Garcia-Lopez MA, Sancho D, Sanchez-Madrid F, Marazuela M (2001) Thyrocytes from autoimmune thyroid disorder produce the chemokine IP-10 and Mig and attract CXCR3+ lymphocytes. J Clin Endocrinol Metab 86:50085016

8. Nicoletti F, Conget I, Di Marco R et al. (2001) Serum levels of the interferon- $\gamma$-inducing cytokine interleukin-18 are increased inindividuals at high risk of developing Type I diabetes. Diabetologia 44:309-311

9. Shimada A, Morimoto J, Kodama K et al. (2001) Elevated serum IP-10 levels observed in type 1 diabetes. Diabetes Care 24:510-515

10. Hussain MJ, Peakman M, Gallati H et al. (1996) Elevated serum levels of macrophage-derived cytokines precede and accompany the onset of IDDM. Diabetologia 39:60-69 